to enlarging our experience of the behaviour of diseased processes, history inspires due admiration for our great predecessors, it teaches humility, it stimulates the spirit of investigation and the search after truth, and by bringing us in contact with literature and the arts it makes us worthier members of a cultured profession.

The work is planned on the lines of the usual text-book of medicine, the historical references being grouped under the headings "The Infectious Diseases," "Diseases of Metabolism," "Diseases of the Circulatory System," "Respiratory Diseases," "Blood Diseases," "Deficiency Diseases," etc. Extracts arranged in chronological order, from the earliest and original descriptions of the individual diseases naturally comprise the bulk of the text, but this is amplified by short biographical sketches, portraits of the pioneers, reproductions of the title pages of famous pamphlets and books and of illustrations in the original publications.

To our mind no more perfect method of stimulating interest in the subject and of supplying the information could be imagined and places the medical profession under a deep debt of gratitude to the distinguished author, Prof. Major.

\section{BROMPTON HOSPITAL REPORTS.}

Vol. VII. 1938. Gale \& Polden, Ltd., Aldershot. Price 5s. 0d.

Although for the most part the articles in this volume are reprints of papers which have already had wide publicity, and perhaps greater permanency than the present volume will provide, by their appearance in the best known and thus usually referred to medical journals, the compilation serves the useful purpose of demonstrating by a process of mass dosage the good work in clinical investigation which is being carried on within the walls of the Brompton Hospital.

The first three papers of the series are from the pen of the late Dr. L. S. T. Burrell, one of the best known members of Brompton's staff and whose loss the editors record in their preface. These articles are on "Creeping Tuberculosis," "Abscess of the Lung," and "The Classification of the Pneumonias." Other valuable reprints are those" on "The Etiology of Bronchiectasis" and "The Pathogenesis of Bronchiectasis" by Drs. Lee Lander and Maurice Davidson although in our opinion a more comprehensive and serviceable treatment of the subject would have resulted from combining the two articles in one. Other reprints are "Radiology and The Surgery of Inflammatory Lesions of the Lungs" by Dr. Sparks, "The Prevention of Lesion Ailments" and "Cardiac Emergencies" by Dr. Clifford Hoyle, and "Obstruction of the Trachea" by Mr. Ormerod.

A specially interesting and comprehensive and previously unpublished article is by Mr. Brock on "Thoracoscopy and Cauterization of Adhesions," which is based on the experience obtained from 442 consecutive operations. And, as has been the custom in these Reports, the volume concludes with a statistical analysis of the clinical material observed during the year-in the present instance during 1937.

\section{SHOCK AND RELATED CAPILLARY PHENOMENA.}

By Virgil H. Moon. Oxford University Press. 1938. Price 21s. Od.

During the Great War a co-operative investigation of shock was undertaken by interested surgeons and physiologists, but unfortunately morphological pathologists were not co-opted. Such a defect has been corrected by the researches of a pathologist, Virgil Moon, in his book "Shock and related capillary phenomena".

The various theories concerning shock are critically examined-nervous exhaustion, acapnia, fat embolism, decreased alkali reserve, and traumatic toxæmia, and the defects in each outlined.

Moon offers evidence which suggests that the capillary endothelium itself is contractile, and that the real control of the opening and closing of the normal capillary loops is a metabolic one from the presence of metabolites and lack of oxygen on the one hand, and from hormonal substances (pituitary) on the other. They are also highly sensitive to other influences such as changes in temperature, light, $\mathrm{H}$-ion concentration, and by a wide variety of organic and inorganic substances. Hence either capillaries or arteries may contract or dilate independently.

Moon defines shock as a circulatory deficiency, neither cardiac nor vasomotor in origin, characterised by a decreased blood volume, decreased cardiac output (reduced volume flow) and by increased concentration of the blood. This hæmo-concentration is the earliest detectable manifestation of shock as well as the most accurate index of its severity. In the development of shock, two major factors are operative; (a) atony and dilatation of the capillaries and venules in extensive visceral areas, and (b) anoxia. This relationship constitutes a self-perpetuating mechanism which operates as a vicious circle.

Experiments show that wheal formation does not result from differences between intra- and extra-vascular pressure but from the liberation of a diffusible substance of a histamine-like nature. For the urticaria of protein sensitivity Moon proposes an alternative explanation to this "triple response" of Lewis. He suggests that not only the tissue cells but also the capillary endothelial cells are sensitized and consequently are directly injured by contact with the antigen and not necessarily injured indirectly through the agency of a substance released by the tissue cells in response to the injury. Moon concludes that the development of skin wheals, tissue inflammation and systemic circulatory failure in anaphylaxis is significantly similar to wheals, inflammation and shock following trauma and other injuries to living tissues. He considers the fundamental capillary reactions in these respective phenomena identical. But unfortunately in 